\title{
Incidences socio-économiques et sanitaires de la mise en valeur durable des bas-fonds de la commune de Zagnanado (département du Zou, Bénin)
}

\author{
B. Mathieu HOUNSOU ${ }^{*}$, A. C. Sandra BOKO ${ }^{1}$, S. Prudence BADJITO ${ }^{2}$, \\ M. Voltaire ALOFA ${ }^{1}$ et K. Euloge AGBOSSOU ${ }^{1}$
}

${ }^{1}$ Laboratoire d'Hydraulique et de Maîtrise de l'Eau (LHME/INE/UAC), Bénin. ${ }^{2}$ Cellule Bas-Fonds de la Direction du Génie Rural (CBF/DGR), Bénin.

*Auteur correspondant ; E-mail : hounsoumat@gmail.com

\section{RÉSUMÉ}

La mise en valeur des terres humides entraîne une amélioration de la production agricole, mais est parfois la cause des problèmes de santé chez certains exploitants. L'étude faite sur les bas-fonds de la commune de Zagnanado précisément ceux de Dovi-Zounnou, de klobo-gbadji, de Bamè et de Zonmon porte sur la caractérisation socio-sanitaire des ménages agricoles dans le cadre d'une exploitation durable des zones humides. Les conditions naturelles qui déterminent ces écosystèmes font de ces derniers, des sites qui font face aux situations sanitaires difficiles et contraignantes du fait de certaines affections (paludisme, gastro-entérite, cholera, l'ulcère de Buruli, la dracunculose, etc....) liées à l'environnement. Il résulte de ces affections, des pertes de jours d'activités dont les conséquences au plan économique sont importantes. Dans la présente étude, des entretiens de groupe semi-structurés ont été tenus dans (04) villages exploitant les bas-fonds sur 10 que compte la Commune. De plus, un échantillonnage raisonné de 304 ménages agricoles soit $62 \%$ a été constitué et prenant en compte les ménages exclusifs des bas-fonds, les ménages mixtes et les ménages exclusifs des plateaux. L'estimation de la perte financière a été réalisée en fonction du nombre de jours de travail perdu, des coûts de traitement et les dépenses en main d'œuvre. Les résultats ont montré que, i) l'exploitation agricole des bas-fonds a amélioré la production maraîchère (piment) et le revenu agricole des exploitants comparativement aux ménages exploitant exclusivement les plateaux ; ii) la prévalence du paludisme, des gastro-entérites, du choléra, de l'ulcère de Buruli et de la dracunculose est plus élevée dans les ménages exploitant les bas-fonds $(15,75 \%)$ comparativement aux ménages exploitant exclusivement les plateaux (10,75\%). De plus, ces maladies entraînent des pertes financières liées aux pertes de jours d'activité considérables pour les ménages exploitant exclusivement les bas-fonds qui peuvent s'évaluer à plus de 180000 FCFA par hectare pour la campagne. Tout ceci montre que l'exploitation des zones inondables constitue une stratégie de lutte contre la pauvreté mais elle a un effet sur la santé des populations.

(C) 2020 International Formulae Group. All rights reserved.

Mots clés : Bas-fonds, ménages agricoles, écosystème, maladies hydriques, Zagnanado.

\section{Socio-economic and health impacts of the sustainable development of inlands valleys in Zagnanado municipality (department of Zou, Benin)}

\begin{abstract}
Wetland development leads to improved agricultural production, but is sometimes the cause of health problems for some farmers. The study of the low lands areas of the municipality of Zagnanado, specifically those
\end{abstract}


of Dovi-Zounnou, klobo-gbadji, Bamè and Zonmon, focuses on the socio-health characterization of agricultural households in the context of sustainable exploitation of wetlands. The natural conditions that determine these ecosystems make them sites that face difficult and restrictive health situations due to certain conditions (malaria, gastroenteritis, cholera, Buruli ulcer, dracunculosis, etc.) related to the environment. As a result of these conditions, the loss of activity days, the economic consequences of which are significant. In this study, semistructured group interviews were held in 4 villages of 10 exploiting the valley areas in the Commune. In addition, a reasoned sample of 304 agricultural households, or $62 \%$, was compiled, taking into account exclusive low lands households, mixed households and exclusive up lands households. The estimation of the financial loss was based on the number of working days lost, processing costs and labour costs. The results showed that, (i) low lands farming improved vegetable production (pepper) and farmers' farm income compared to households exclusively operating on up lands; (ii) the prevalence of malaria, gastro-enteritis, cholera, Buruli ulcer and dracunculosis is higher in low lands households (15.75\%) compared to households operating exclusively on the up lands area $(10.75 \%)$. In addition, these diseases result in financial losses related to the loss of significant working days for households operating exclusively in the low lands, which can amount to more than 180,000 CFA francs per campaign. In conclusion, the exploitation of flood zones is a strategy to fight poverty but it has an effect on the health of populations.

(C) 2020 International Formulae Group. All rights reserved.

Keywords: Valleys, agricultural households, ecosystems, water diseases, Zagnanado.

\section{INTRODUCTION}

Dans son rapport sur l'insécurité alimentaire dans le monde en 2015, l'Organisation des Nations Unies pour l'Alimentation et l'Agriculture (FAO) observe que, 795 millions de personnes sont victimes de sous-alimentation, soit une diminution de 167 millions de personnes sur ces dix dernières années. Le fléchissement est plus net dans les régions en développement, en dépit d'une forte croissance de la population. Les progrès ont été freinés par une croissance économique ralentie et moins inclusive, ainsi que par l'instabilité politique dans certaines régions en développement, notamment en Afrique centrale et en Asie de l'Ouest selon un rapport de la FAO de 2015. Selon le même rapport, dans la plupart des régions en développement, le secteur agricole est celui où le risque de pauvreté au travail est le plus élevé ; soit huit sur dix environ des travailleurs touchés par la pauvreté ont des emplois vulnérables dans l'économie informelle, particulièrement dans l'agriculture.

Mais l'agriculture est capable à elle seule d'amorcer la croissance dans les pays où elle représente une forte part du PIB selon le même rapport de la FAO. Au Bénin par exemple, l'économie est dépendante du secteur agricole pour environ $70 \%$ des emplois et $32 \%$ du Produit Intérieur Brut (PIB) selon un rapport $\mathrm{du}$ Fonds International de Développement Agricole (FIDA) en 2015.

Selon Biaou (2012), les conséquences de la forte pression agro démographique et de la dégradation des terres sur le plateau, la disparition des endémies et les effets néfastes des aléas climatiques, tout en rendant aléatoires les cultures ont amené les producteurs à s'adonner à l'exploitation des bas-fonds jadis marginalisés par ce secteur. Cette réalité conduit les paysans vers ces terres non encore cultivées pour leur mise en valeur. C'est dans ce cadre que les producteurs s'investissent dans l'exploitation des écosystèmes humides tels que les bas-fonds très fertiles qui offrent plusieurs possibilités agricoles et piscicoles. Bien que le développement de l'agriculture dans les bas-fonds soit essentiel pour augmenter la productivité agricole partout en Afrique subsaharienne, les effets secondaires négatifs de cette intensification de l'agriculture sont aussi considérables tant sur les sols, que sur les ressources en eau et sur la santé des exploitants. Ainsi, de nombreux problèmes écologiques liés à l'agriculture vont rester préoccupants au cours des 30 prochaines années. En effet, l'agriculture dans ces milieux 
pourrait avoir des répercussions profondes tels que la modification de l'hydrologie des cours d'eau et la réduction des taux de recharge des eaux souterraines, l'envasement et l'altération des écosystèmes connexes sans omettre l'introduction d'espèces végétales envahissantes. De même, la monoculture et l'intensification agricole accroissent le risque écologique en favorisant la baisse de la fertilité, l'érosion des sols et la diminution de la biodiversité (Malezieux et Moustier, 2005). Par ailleurs, l'eau facilite le transport d'agents pathogènes et de contaminants chimiques de l'environnement jusqu'à la chaîne alimentaire, avec une incidence sur la sécurité alimentaire et la santé publique. Le Bénin dispose de plusieurs bas-fonds auxquels s'intéressent davantage les paysans face à la dégradation sans précédent des terres du plateau (Hounsou, 2003). Mais, les aspects socio-sanitaires de leur exploitation ont très peu fait l'objet de recherche bien qu'ils soient déterminants dans les stratégies de conservation et de gestion rationnelle durable de ces milieux.

Dans les zones humides du Bénin, particulièrement celles de Zagnanado, les Maladies Liées à l'Eau (MLE) telles que le paludisme (grave, simple confirmé et non confirmé), les infections gastro-entérites (IGE), la shiguellose, le choléra, la diarrhée fébrile ou avec déshydratation, la dracunculose, l'ulcère de Buruli sévissent fréquemment pour ne citer que celles-là.

Cette étude vise à évaluer d'une part, les risques socio-sanitaires qu'encourent les ménages qui exploitent les bas-fonds et d'autre part, les coûts directs et indirects liés à ces risques sanitaires, tout ceci comparativement aux ménages exploitant strictement les plateaux.

\section{MATERIEL ET METHODES}

\section{Présentation de la zone d'étude}

La commune de Zagnanado est située sur le plateau de Zagnanado, le plus petit des plateaux au nord de la dépression de la Lama au Bénin ; elle est située entre $7^{\circ}$ et $7^{\circ} 30^{\prime}$ de latitude nord et entre $2^{\circ} 15^{\prime}$ et $2^{\circ} 30^{\prime}$ de longitude est et se retrouve dans le département du Zou. La superficie totale de la Commune est de $750 \mathrm{~km}^{2}$. Elle est limitée au Nord par la commune de Dassa-Zoumè ; au Sud par celles de Zogbodomè et de Ouinhi ; à l'Est par les communes de Kétou et d'AdjaOuèrè ; à l'Ouest par celles de Covè, Za-Kpota et Djidja. La Commune se retrouve partagée sur deux bassins versants. Près des trois quarts de sa superficie $\left(433 \mathrm{~km}^{2}\right)$ sont drainés vers le fleuve Ouémé et le reste $\left(166 \mathrm{~km}^{2}\right)$ s'écoule vers la rivière Zou. Le rythme pluviométrique est de type bimodal avec des précipitations moyennes mensuelles de $985 \mathrm{~mm}$ d'eau, réparties sur 74 jours en moyenne. Les températures annuelles oscillent entre $19,9^{\circ} \mathrm{C}$ et $34^{\circ} \mathrm{C}$. La Commune jouit d'un climat de type intermédiaire à cheval entre le climat subéquatorial maritime et le climat soudanoguinéen. Les sols sont généralement de types hydromorphes au sud, ferrugineux au nord et ferralitiques au centre. Les physionomies végétales rencontrées sont les savanes arborées, des forêts denses sèches, semidécidues, des forêts claires, des savanes boisées, des savanes arbustives (Mama, 2013).

Pour réaliser cette étude, la méthodologie adoptée s'est basée sur la recherche documentaire, la collecte des informations épidémiologiques au niveau des centres de santé fréquentés par les exploitants de la zone, les enquêtes menées sur le terrain à travers les localités où les bas-fonds sont exploités et des observations participantes sur les sites.

\section{Collecte des données}

\section{Recherche documentaire et collecte d'informations aux centres de santé}

Comme tout travail scientifique, des informations et données ont été collectées à travers des structures de recherches, les services techniques et sur internet. Des données sur la santé des populations sont collectées au niveau de trois (03) centres de santé et d'un hôpital qui sont plus fréquentés par les ménages du village et en particulier les exploitants du bas-fond. 


\section{Echantillonnage des ménages pour les enquêtes}

L'unité d'échantillonnage est le ménage agricole au sein de l'ensemble des ménages des villages sélectionnés dans la zone d'étude. La technique d'échantillonnage raisonnée a été adoptée et elle a pris en compte le Groupe de Ménages Spécifiques des Bas-fonds (GMSB), le Groupe de Ménages Spécifiques des Plateaux (GMSP) et le Groupe de Ménages Mixtes (GMM). Dans la suite de ce document, seules les abréviations des groupes de ménages seront utilisées et tous les bas-fonds identifiés dans la Commune ont été pris en compte. Plusieurs villages pourraient se retrouver à exploiter un bas-fond. Alors, seuls les villages possédant les trois groupes de ménages ciblés (GMSB, GMM et GMSP) ont été retenus. Dans chaque village, seuls les ménages ayant une expérience agricole d'au moins 5 ans dans l'exploitation des bas-fonds ont été retenus aléatoirement. Le taux d'échantillonnage appliqué est de $62 \%$ soit un échantillon de 188 sur un total de 304 ménages agricoles pour toute la zone. Le Tableau 1 (Annexes) présente le nombre de ménages enquêtés par bas-fond et par groupe de ménage.

\section{Collecte des données}

Les caractéristiques économiques et sanitaires des ménages sont des données moyennes des cinq (05) années de campagnes agricoles (2007-2011), collectées à l'aide d'un guide d'entretien et d'un questionnaire administré dans les trois (03) groupes de ménages constitués. Ainsi, la production maraîchère en grande saison pluvieuse, la superficie allouée au maïs, le prix de vente et le coût de production, pour chaque année, le nombre d'actifs souffrant de la maladie « $\mathrm{i}$ » dans le ménage ; le nombre d'actifs agricoles du ménage ; le nombre de jours perdus avant la consultation ; le nombre de jours de traitement hors ménage; le nombre de jours de convalescence ; le nombre de jours perdus par les membres du ménage pour cause de visite au malade ; le nombre de jours d'entraide sociale ou de main d'œuvre salariale agricole ; la valorisation monétaire de la journée de travail
; les investissements réalisés en agriculture du fait de la perte de jours d'activités et les dépenses de santé sont des données collectées. Par ailleurs, dans la Commune, les statistiques sanitaires de 2007 à 2011 ont été consultées dans les centres de santé afin de s'assurer de l'existence des Maladies Liées à l'Eau (MLE).

Pour cette étude, la spéculation choisie est le piment grâce à ces atouts définis par les exploitants : la courte période de production en saison sèche (75-90 jours), l'accès facile à moindre coût des semences de meilleures variétés (piment antillais en forme de lanterne), le taux élevé de rendement à la récolte, sa richesse en vitamine $\mathrm{C}$ très appréciée; et surtout, il est consommé dans presque tous les repas dans la localité. Il est aussi transformé en poudre, embouteillé ou exporté au-delà du pays. Son fruit dans les bas-fonds est bien dressé de 4-6 cm de long, allongé et pointu, saint de couleur vif à maturité. Cette spéculation limite beaucoup les travaux d'entretien et de chasse des oiseaux qui ne s'y approchent pas trop mais par contre sucent le riz en cours de développement et détruisent les champs de maïs malgré les différentes initiatives de protection des champs. Les bénéfices sont doubles de ceux des spéculations du riz et du maïs lorsqu'on les compare. Pour ces derniers, on intègre tellement de coûts élevés d'intrants agricoles liés à la production. Aussi, l'effort physique déployé est très relaxe et le temps de travail limité par rapport au riz et au maïs cultivés dans les mêmes conditions de bas-fonds.

\section{Traitement des données}

Les données ont été traitées dans un esprit de comparaison quantitative entre les trois (03) groupes de ménages. Le logiciel statistique Minitab 14 a été utilisé pour l'analyse de variance (ANOVA) à un facteur afin de comparer les données moyennes entre les MSB ; les MM et les MSP. Quand ce test révèle une différence significative entre les trois (03) groupes de ménage, alors le test $\mathrm{t}$ de Student à deux échantillons est utilisé pour détecter les types de ménage différents. Les 
formules utilisées pour les différentes variables sont :

\section{Moyenne arithmétique}

Dans chaque groupe de ménages, la production moyenne de riz, de piment et de maïs par ménage, le revenu moyen brut par ménage, le coût moyen de production par ménage et la marge moyenne brute par ménage ont été déterminés suivant la formule de la moyenne arithmétique de Dagnelie (1973).

$x i=\frac{1}{n} \sum_{i=1}^{n} x i$

$x i$ : valeur observée de la variable $\mathrm{X}$ au niveau du ménage $\mathrm{i}$

$n:$ nombre de ménages constituant le groupe de ménages

Estimation du Revenu brut (Rbi) de chaque ménage (FCFA)

Le Revenu brut de chaque ménage a été obtenu en multipliant le prix de vente du produit par la quantité produite. Ceci concerne ici uniquement la production du piment.

$\boldsymbol{R} \boldsymbol{b i}=\left(\boldsymbol{P} \boldsymbol{i}^{*} \boldsymbol{P m}\right)$

$P i$ : Production de la spéculation triée (piment) du ménage i

$P m$ : Prix moyen de vente de la spéculation (piment trié $(200 \mathrm{f} / \mathrm{Kg})$ )

Estimation du Coût de production (Cpi) de chaque ménage (FCFA)

Le coût de production du piment de chaque ménage est la dépense totale prenant en compte les opérations agricoles tels que le défrichement/désherbage, la préparation de sol, le semis/repiquage, la fertilisation, l'entretien, la récolte et l'achat d'engrais et de semence.

$\mathbf{C p i}=\sum_{i=1}^{n} \boldsymbol{C v}$

$C v$ : Dépense liée à une opération agricole donnée.

Estimation de la Marge brute (Mbi) de chaque ménage (FCFA)

La marge brute de chaque ménage a été obtenue par une formule citée par Kinkingninhoun (2003).

$M b i=(R b i-C p i)$
$R b i$ : Revenu brut du ménage $\mathrm{i}$

Cpi : Coût de production supporté par le ménage $\mathrm{i}$

Estimation du rendement de la production maraîchère (Ri) de chaque ménage (tonne/hectare)

Le rendement du piment au niveau de chaque ménage a été déterminé suivant la formule proposée par Aho et Kossou (1997).

\section{$\mathbf{R i}=\boldsymbol{P i} / \boldsymbol{S i}$}

$P i$ : Production sélectionnée du ménage i

$S i$ : Superficie consacrée à la culture par le ménage $\mathrm{i}$

Les données sanitaires moyennes ont été calculées pour chaque ménage (MSB ; MM et MSP). Les formules utilisées par Azonhè (2009) ont servi pour les données sanitaires moyennes de chaque ménage :

- Prévalence annuelle de la maladie i (Pi) au niveau du ménage

$\operatorname{Pi}(\%)=\mathrm{Ni} / \mathrm{N}$

$\mathrm{Pi}$ : Prévalence annuelle de la maladie i au niveau du ménage ;

$\mathrm{Ni}$ : Nombre moyen annuel de cas de maladie i au niveau des actifs agricoles du ménage ;

$\mathrm{N}$ : Nombre moyen annuel d'actifs agricoles du ménage.

La prévalence suit une loi binomiale, il a été procédé à une transformation angulaire suivant la formule $\mathrm{Yi}=2 \operatorname{arcSin} \mathrm{Pi}$ (Dagnelie, 1973).

- Perte financière annuelle due à la maladie i (Pfi) au niveau du ménage

Pfi $($ fcfa $)=($ Pwi $*$ Vejt $)+$ Ia + Is

Pfi : Perte financière annuelle due à la maladie i au niveau du ménage ;

Pwi : Perte annuelle de temps de travail due à la maladie i au niveau du ménage ;

Vejt : Valorisation monétaire de la journée de travail ;

Ia : Investissements moyens réalisés en agriculture dus à la perte de jours d'activités ; Is : Dépenses moyennes de santé 
Tableau 1 : Taille de ménages enquêtés par bas-fond et par groupe de ménage.

\begin{tabular}{|c|c|c|c|c|c|c|c|c|}
\hline \multirow[b]{2}{*}{$\mathbf{n}^{\circ}$} & \multirow{2}{*}{$\begin{array}{c}\text { Bas-fonds } \\
\text { retenus }\end{array}$} & \multirow{2}{*}{$\begin{array}{l}\text { Villages } \\
\text { retenus }\end{array}$} & \multirow{2}{*}{$\begin{array}{c}\text { Nombre de } \\
\text { ménages } \\
\text { agricoles }\end{array}$} & \multicolumn{4}{|c|}{ Ménages enquêtés } & \multirow[b]{2}{*}{$\%$} \\
\hline & & & & $\begin{array}{c}\text { GMS } \\
\text { B }\end{array}$ & GMM & $\begin{array}{c}\text { GMS } \\
\text { P }\end{array}$ & Total & \\
\hline 1 & Dovi-zounnou & $\begin{array}{l}\text { Dovi- } \\
\text { zounnou }\end{array}$ & 107 & 2 & 43 & 21 & 66 & 62 \\
\hline 2 & Klobo & Klobo & 32 & - & 13 & 6 & 19 & 59 \\
\hline 3 & Bamè & \multirow{2}{*}{ Bamè } & 52 & 1 & 21 & 10 & 32 & 62 \\
\hline 4 & Site des jeunes & & 33 & 1 & 13 & 7 & 21 & 64 \\
\hline 5 & Zoumon & Zoumon 1 & 50 & 1 & 20 & 10 & 31 & 62 \\
\hline 6 & Zonmon & Zonmon 2 & 30 & 1 & 12 & 6 & 19 & 63 \\
\hline & Total & 5 & 304 & 6 & 122 & 60 & 188 & 62 \\
\hline
\end{tabular}

GMSB : Groupe de Ménage Spécifiquement Bas-Fonds ; GMM : Groupe de Ménage Mixte ;

GMSP : Groupe de Ménage Spécifiquement Plateau.

\section{RÉSULTATS}

\section{Caractérisation socio-économique}

Analyse des retombées de la mise en valeur des bas-fonds sur la production agricole

Dans le bas-fond, les principales cultures pratiquées sont le riz et les cultures maraîchères, avec le riz comme principale spéculation. Mais cette dernière n'est pas pratiquée sur le plateau. Ainsi, pour évaluer l'impact de l'exploitation agricole des basfonds, nous avons retenu le piment qui est une spéculation produite à la fois sur les deux types de terres. Il s'agira d'analyser les rendements afin de comparer la fertilité et les potentialités du bas-fond à celles des terres des plateaux. La figure 1 (Annexes) indique les statistiques annuelles de production (production et superficie) des cultures de piment obtenues par groupe de ménages (GMSB, GMM et GMSP) dans la zone d'étude.

De l'analyse de cette Figure 1, il ressort que le GMSB est plus productif que le GMSP car la production moyenne obtenue dans les bas-fonds est supérieure à celle de GMSP. Pour le rendement moyen obtenu au niveau du basfond, cela avoisine le double de celui obtenu sur le plateau. Aussi constate-t-on que même le rendement minimum calculé sur le bas-fond dépasse largement le rendement maximal de cette spéculation sur les plateaux. Les terres du bas-fond sont donc plus productives que celles des plateaux. Il est observé également que, les productions agricoles les plus élevées sont obtenues par les ménages exclusifs des basfonds $(5,068 \pm 2,244 \mathrm{t})$ et les ménages mixtes $(5,347 \pm 1,468$ t) contrairement aux ménages exclusifs des plateaux qui ont la production agricole la plus faible $(3,850 \pm 1,030 \mathrm{t})$ malgré la fertilisation chimique adoptée. Ensuite, bien que les superficies emblavées dans les basfonds soient plus faibles (5-10 ha) comparativement à celles des plateaux (8-14 ha), de fortes productions agricoles sont obtenues, ce qui montre la forte influence du rendement moyen $(0,511 \mathrm{t} / \mathrm{ha})$ qui avoisine le double de celui du plateau $(0,35 \mathrm{t} / \mathrm{ha})$. Mais la question qui se pose est de savoir, si la différence est réellement significative ? Le test statistique de comparaison de moyenne effectué, montre que cette différence de rendement est hautement significative au seuil de $5 \%$ et même au seuil de $1 \%,(P=0,000)$.

Des résultats ainsi obtenus, il ressort que, malgré le caractère saisonnier des cours d'eau qui alimentent les différents bas-fonds, les potentialités du bas-fond telles que sa bonne fertilité, peuvent permettre d'augmenter significativement les rendements et de surcroît de diversifier les cultures. En effet, outre les spéculations produites sur les terres du plateau, le bas-fond permet également de produire plus facilement d'autres cultures comme la canne à sucre, le taro, la banane et les produits maraîchers qui sont toutes exigeantes en eau. 


\section{Retombées de l'exploitation sur le revenu des ménages}

Il s'agit ici d'évaluer l'impact de la mise en valeur du bas-fond en gain supplémentaire de revenu pour les ménages et si elle mérite d'être encouragée. Signalons qu'avant les différents aménagements, les bas-fonds n'étaient que faiblement exploités. Leur mise en valeur ne se résumait qu'à une production très limitée de légumes sur les versants. Ainsi, nous ne disposons pratiquement d'aucune donnée économique relative à la mise en valeur de ces sites avant aménagement. Pour cela, nous proposons dans cette étude, de voir si actuellement, l'aménagement, à travers le degré de maîtrise de l'eau et les nouvelles spéculations, permet de pratiquer ou d'améliorer le revenu des exploitants. La Figure 2 montre la performance économique de la culture pour une campagne.

Cette Figure 2 révèle une différence significative entre les marges brutes par hectare de production pour une campagne par groupe de ménage. Il se dégage que la mise en valeur du bas-fond génère aux exploitants plus de revenus que celle des plateaux. Qu'il exploite ou non les plateaux, le revenu moyen d'un exploitant du bas-fond au cours d'une campagne agricole est nettement supérieur à celui d'un exploitant du plateau. Aussi, cette différence semble s'observer entre les marges brutes moyennes tirées uniquement du basfond et celles tirées de l'exploitation mixte. Considérant le bas-fond et le plateau d'une part et le plateau et la zone mixte d'autre part, les tests statistiques respectifs révèlent dans chacun des cas que la différence est hautement significative au seuil de $1 \%$. Ainsi, tout exploitant du bas-fond, qu'il exploite ou pas le plateau a un revenu nettement supérieur à celui de ceux qui exploitent exclusivement les plateaux. S'agissant du bas-fond et de la zone mixte, le test statistique réalisé révèle que la différence n'est pas significative au seuil de $5 \%$ $(\mathrm{P}=0,411)$; Autrement dit, l'exploitation exclusive de bas-fond ne génère pas forcément plus de revenus qu'une exploitation mixte. Cela se justifie par trois raisons essentielles : le phénomène d'inondation qui limite la production sur 4 mois au cours de l'année alors que sur les plateaux cela se fait à plein temps tant qu'il y a de l'eau pour arroser; aussi l'entraide sur les plateaux est plus importante comparativement aux bas-fonds où c'est la main d'œuvre salariée qui prédomine. Les dépenses liées au coût de celle-ci sont donc énormément réduites sur les plateaux ; enfin les exploitants mixtes délaissent souvent leurs cultures pour entretenir et récolter les vivriers sur les plateaux. Ce qui fait que les travaux d'entretiens notamment le traitement phytosanitaire et la fumure sont souvent négligés sur les parcelles de bas-fonds et ceci provoque, par conséquent, la pourriture des plants. Ce résultat montre que le maraîchage à elle seule améliore nettement le revenu des ménages qui s'y adonnent. En somme, la mise en valeur du bas-fond à travers le maraîchage sur bas-fond, améliore significativement le revenu des exploitants. Elle génère moyennement le double des revenus de n'importe quelles autres cultures, tirés des plateaux. Il est donc très avantageux aux exploitants de cultiver dans les bas-fonds.

Toutefois, il est nécessaire de signaler qu'il est plus profitable aux exploitants des basfonds de procéder à une exploitation mixte (rizmaraîchage) et aux exploitants exclusifs (maïsmaraîchage). Bien que ceci ne soit pas sans risque (notamment les retards dans la campagne et les mauvais entretiens des cultures faute de temps, etc..), les avantages sont énormes. Citons par exemple la disponibilité de vivres (maïs, fruit et légumes) notamment ajoutés au riz qui permet aux exploitants mixtes de pouvoir traverser plus facilement les périodes de soudure et de réduire considérablement les dépenses liées à l'alimentation.

De l'analyse des données socioéconomiques, on retient que, sur une année, le paysan qui exploite le bas-fond pourrait obtenir environ $511 \mathrm{~kg} / \mathrm{ha}$ de piment par exemple alors que sur les plateaux, il n'obtiendrait que 350 $\mathrm{kg} / \mathrm{ha}$. 


\section{Caractérisation sanitaire}

La situation sanitaire de la zone d'étude est essentiellement basée sur les relevés épidémiologiques des centres de santé d'Agonli-Houégbo (CSAH), de Dovi-Dovè (CSDD), de Zagnanado (CSZ) et l'Hôpital Confessionnel Gbèmontin (Figure 3).

De la Figure 3, il ressort que le paludisme (PAL) est la première cause de consultation avec un taux annuel de cas élevé (43\%). Les affections gastro-entérites (AGE) viennent ensuite avec des taux annuels différents selon les centres. Enfin viennent le cholera (CHO), l'ulcère de Buruli (UB) et la dracunculose (DRA) qui font très peu l'objet de consultations dans les centres.

La prévalence des maladies est plus élevée au niveau de la population fréquentant les centres sanitaires, ce qui pose le problème de fréquentation des formations sanitaires qui est de $40 \%$ dans la zone d'étude selon les registres épidémiologiques des centres de santé. Le paludisme et les gastro-entérites sont les premières maladies touchant les actifs agricoles avec une fréquence de 1 à 3 fois par an surtout au niveau des ménages exploitant les bas-fonds. Le travail dans les bas-fonds, la proximité bas-fonds/habitations et l'efficacité partielle des stratégies préventives sont les causes de cette prévalence du paludisme. La consommation des eaux des cours d'eau pendant des travaux champêtres et le manque d'hygiène alimentaire expliquent la prévalence des gastro-entérites malgré l'existence des forages. Par ailleurs, le choléra et l'ulcère de Buruli sont les maladies les moins fréquentes mais les plus invalidantes. Elles touchent entre $5-16 \%$ des actifs agricoles d'un ménage au cours d'une année avec une forte prévalence au niveau des ménages exploitant les bas-fonds. Le contact fréquent avec les eaux des bas-fonds pour des raisons agricoles ou autres raisons sont à l'origine de ces maladies. Les analyses de variance montrent une différence significative entre les proportions des cas d'ulcère de Buruli des trois groupes au seuil de $5 \%$ (respectivement $\mathrm{P}=0,007$ ) contrairement au résultat du test d'analyse de variance sur le paludisme $(\mathrm{P}=0,071)$. Les analyses de variance indiquent qu'il n'y a pas une différence significative entre les proportions des cas de gastro-entérites, du choléra et de la dracunculose exclusivement pour les exploitants des bas-fonds et celle des ménages mixtes et ceci au seuil de 5\% (respectivement $\mathrm{P}=0,039 ; \mathrm{P}=0,332 ; \mathrm{P}=0,952$ ). Du point de vue général, l'exploitation agricole des bas-fonds est source de maladies aux ménages spécifiquement d'ulcère de Buruli. Ces moments de maladies entrainent des perturbations dans le travail donc des pertes financières.

\section{Perte financière}

Plusieurs facteurs expliquent la perte financière par ménage et suivant les cas de maladies liées à l'eau. Nous avons les coûts de traitement, de perte de journée de travail et des dépenses en main-d'œuvre. La Figure 4 montre les proportions des manifestations de ces facteurs par ménage suivant les maladies rencontrées.

De cette figure, nous retenons que peu importe le groupe de ménages considéré, l'ulcère de Buruli, la dracunculose et la gastroentérite sont les maladies qui engendrent le plus des coûts et surtout chez les exploitants des bas-fonds. Aussi, le paludisme et le cholera ont aussi une influence non négligeable sur l'économie des ménages surtout les ménages exploitant exclusivement les bas-fonds et les ménages mixtes. Les résultats de l'analyse de variance montrent une différence significative entre les proportions des dépenses des trois groupes au seuil de $5 \% \quad(\mathrm{P}=0,000)$. L'exploitation agricole des bas-fonds est source de maladies, notamment l'ulcère de Buruli, les gastro-entérites et la dracunculose. On en retient donc que l'appartenance à l'une des catégories de ménage influence le niveau de la perte financière. Cette différence est due au fait que la perte financière globale prend en compte plusieurs variables dont les dépenses en santé. Ces dépenses de santé dont celles en agriculture varient largement d'un ménage à un autre et ne sont pas corrélées à la perte de jours d'activités. 


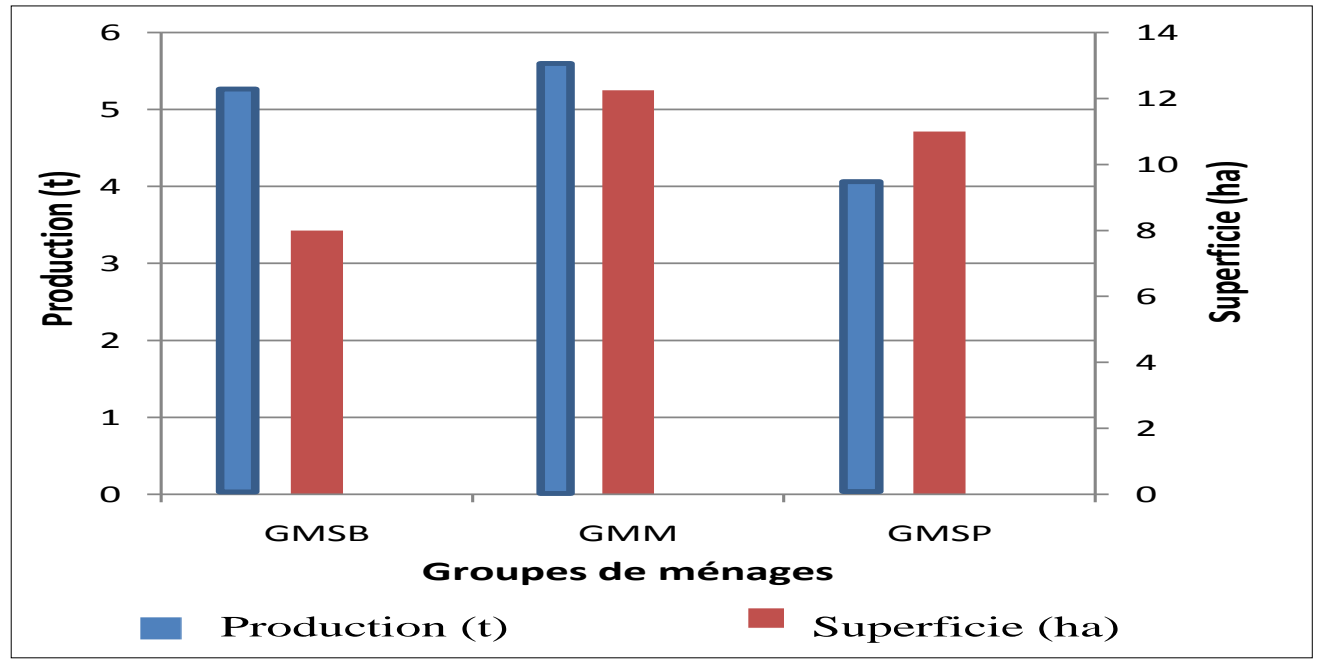

Figure 1 : Productions annuelles obtenues par groupe de ménages dans la zone d'étude.

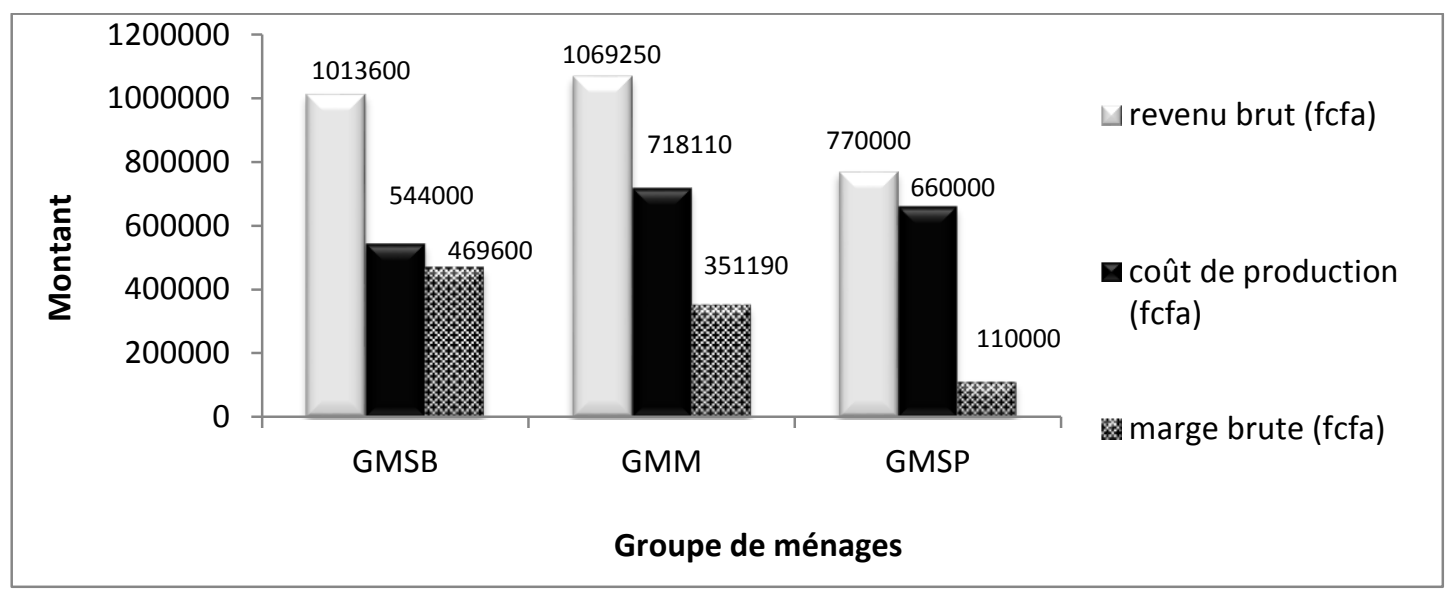

Figure 2 : Performance financière moyenne par groupe de ménage par campagne.

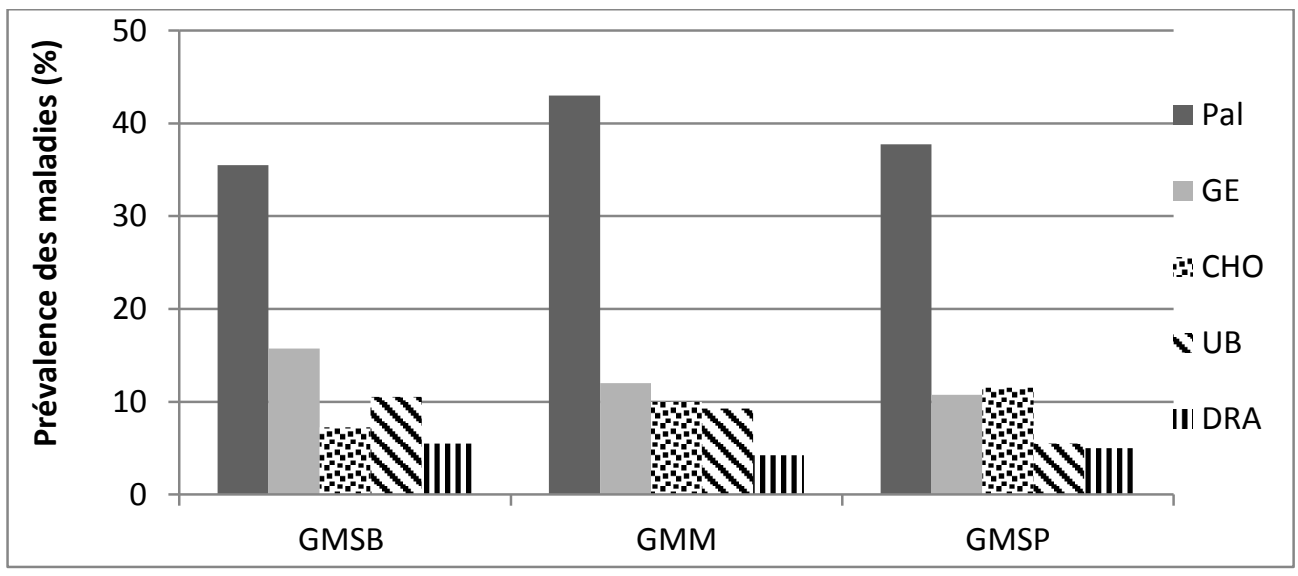

Figure 3 : Prévalence annuelle de maladies liées à l'eau de 2010 à 2015. PAL : Paludisme, GE : Gastro-Entérite, CHO : Cholera ; UB : Ulcère de Buruli ; DRA : Dracunculose. 


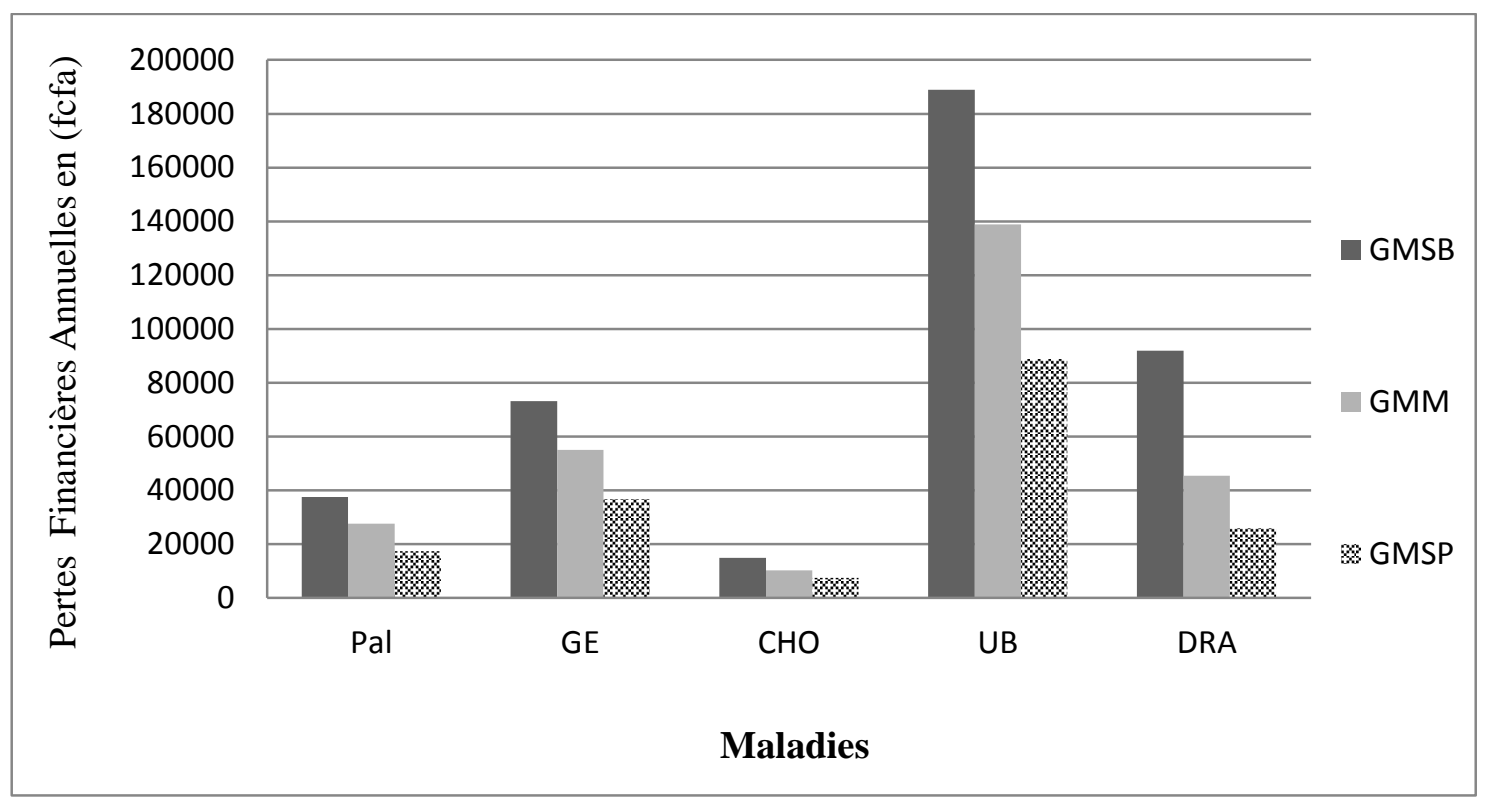

Figure 4 : Pertes financières annuelles par ménage.

\section{DISCUSSION}

\section{Impacts positifs de la mise en valeur des bas-} fonds sur la production et le revenu des ménages

Les rendements des spéculations obtenues dans les bas-fonds sont largement supérieurs à ceux obtenus sur les plateaux et il en est de même pour la production malgré l'utilisation d'engrais chimiques. Il est à noter que les superficies emblavées dans les basfonds sont plus faibles $(5-10$ ha $)$ comparativement à celles des plateaux (8-14 ha).

Aussi, considérant toute la zone d'étude, les résultats montrent que le rendement des cultures maraîchères dans le bas-fond semble toujours être plus élevé que celui du plateau. Les mêmes résultats ont été obtenus par Agbossou et al. (2005) dans les bas-fonds de Logbogba (commune de Covè), où les enquêtes ont montré que les terres de bas-fonds sont plus riches que celles des plateaux. Les résultats obtenus par Vitchoekè (2006) dans le bas-fond Ouantégo de la même Commune, révèlent les mêmes tendances mais obtenus avec le maïs. Il en est de même pour Atidégla et al. (2017) dans les plaines de TodéGbamè (commune de Adjohoun). L'une des causes probables est la différence de fertilité des sols. Abou et al. (2018) affirme dans ce sens que les écosystèmes comme les plaines inondables sont riches, ce qui permet de maximiser les rendements et afin de garantir l'autosuffisance alimentaire.

Sur les bas-fonds, on pourrait avoir le double du rendement obtenu sur les plateaux ; mais on l'avoisine à cause du mauvais entretien des champs de légumes dans le bas-fond et les inondations. En effet, le sarclage qui devait normalement se faire deux, voire trois fois, ne se fait généralement qu'une seule fois dans le bas-fond. Cela influe énormément sur la production. Ces rendements élevés permettent de disposer un surplus de vivres pour la vente et l'autoconsommation. Ces résultats corroborent ceux obtenus dans d'autres études au Bénin, au Mali, au Sénégal et au Vietnam montrant que la production agricole est améliorée dans les zones humides contrairement aux cultures installées sur les 
plateaux (Agbodjogbé, 2008; Jamin et al., 2011 ; Jourdain et al., 2011 ; Badjito, 2012).

En résumé, la mise en valeur des basfonds, augmente donc significativement la production. Elle permet de diversifier les cultures à travers la production de spéculations qui sont difficiles à pratiquer sur les plateaux, moins fertiles et moins humides. Ces rendements s'améliorent au fur et à mesure que les bas-fonds sont aménagés, c'est à dire que les paysans appliquent sur ces écosystèmes humides quelques techniques de la maîtrise de l'eau (diguettes de rétention, canaux de drainage, seuil en béton ou en gabions, etc.).

Cette augmentation significative de la production génère aux exploitants plus de revenus que celle des plateaux. Qu'il exploite ou non les plateaux, le revenu moyen d'un exploitant du bas-fond au cours d'une campagne agricole est nettement supérieur à celui des exploitants du plateau. Toutefois, il est nécessaire de signaler qu'il est plus profitable aux exploitants des bas-fonds de procéder à une exploitation mixte (rizmaraîchage) et aux exploitants exclusifs (maïsmaraîchage). Ces résultats corroborent ceux de Iwikotan et al. (2016) qui ont montré que les produits (riz et légumes) issus de la mise en valeurs des bas-fonds permettent de lutter contre la famine, de réduire la période de soudure et d'avoir des revenus selon les enquêtés. De même Konnon et al. (2014) affirment que le riz à travers l'exploitation des bas-fonds contribue non seulement à la sécurité alimentaire des pays, mais il est aussi pourvoyeur de revenus pour les différents acteurs qui s'y sont engagés.

\section{Incidences sanitaires de la mise en valeur des bas-fonds}

Hormis les avantages importants que génère la mise en valeur de ces zones humides, des affections sont rencontrées au niveau des exploitants. Parmi les différents groupes, l'ulcère de Buruli est une affection spécifique chez les exploitants agricoles des bas-fonds. Par contre d'autres affections comme le paludisme, le choléra sont moins fréquentes mais avec plus de conséquences. Badjito et al. (2014) ont montré qu'en dehors de l'ulcère de Buruli, la grasto-entérite et la bilharziose sont dues à l'exploitation des bas-fonds dans la commune d'Adja-Ouèrè. Mais, des études menées par Orsot et al. (2018) ont montré que la prévalence des infections comme la bilharziose est en relation avec l'âge et le sexe des exploitants. Ainsi plus les personnes travaillant dans ces milieux sont âgées plus le risque d'avoir cette affection est élevé ce qui aura un impact négatif sur les activités.

Iwikotan et al. (2016) à travers leurs études sur l'exploitation des bas-fonds au centre du Bénin (Dassa et Glazoué) ont montré que les problèmes de santé les plus rencontrés sont : le paludisme, les courbatures, la fièvre et l'intertrigo. Ils affirment que ces problèmes de santé perturbent les activités des producteurs et peuvent même les rendre invalides ce qui corrobore nos résultats car ces différentes affections engendrent le plus souvent des dépenses en santé entrainant des pertes financières. En effet, la perte financière est liée généralement à la perte de jours d'activité car toute perturbation dans le déroulement des activités culturales du fait des affections entraîne des pertes financières soit par la non réalisation de l'activité, soit par un investissement en vue de sa réalisation par la main d'œuvre pour les ménages. Dans le même ordre d'idée, Son et al. (2016) ont montré dans la province du Yatenga (Nord du Burkina Faso) que l'utilisation de la main d'œuvre entraine une augmentation des charges de productions compte tenu de sa rareté due à la scolarisation et la migration des jeunes. Ainsi, les pertes de revenus sont effectives à chaque épisode de maladie de manière directe ou indirecte (Azonhè, 2009). L'incapacité de travail du fait de la maladie a donc des conséquences sur le revenu des ménages. Asanté et al. (2004) ont montré que «la valeur de la perte du temps productif au ménage a été estimée à 8,92 dollars US, représentant $56 \%$ du coût total de 
la maladie par épisode. La situation s'aggrave lorsque le ménage connaît plusieurs décès et/ou des décès répétés au cours d'une période donnée ». Audibert (2003) a démontré que " l'effet d'un choc de santé apparaît lorsqu'il est important en ce sens qu'il concerne une proportion élevée de membres actifs du ménage ».

La perte financière n'est donc que l'estimation de ce que les populations auraient pu économiser en ayant été indemnes de ces maladies (le paludisme, la gastro-entérite, le choléra, l'ulcère de Buruli et la dracunculose). On en conclut avec Tizio (2004) que «la mauvaise santé est un facteur de stagnation économique et sociale ». La maladie agit sur le développement humain et sur la croissance par différents canaux : perte de bien-être individuel; impacts sur les conditions d'existence de l'individu malade. Enfin, la mauvaise santé induit des coûts qui, en étant parfois importants, hypothèquent toute velléité d'amélioration de l'autonomie individuelle et de maintien de la croissance économique. En termes de bien être, la maladie occasionne des pertes de possibilité de consommation selon plusieurs canaux : les traitements médicaux sont coûteux et, en l'absence de couverture maladie, les sommes affectées amputent les revenus des ménages. La maladie entraîne également une perte de revenu courant du fait des absences au travail et une perte des revenus futurs du fait de la mort prématurée. Ces pertes de revenu font stagner la consommation individuelle et ne permettent pas d'alimenter la demande globale au niveau macroéconomique. Les évaluations économiques en termes de Disability-Ajusted Life Years (DALYs) concluent qu'un an de vie gagnée en bonne santé est considérablement supérieur à un an de revenus ». La perte financière induite par les pertes de jours d'activités participe aux difficultés de gestion de la maladie chez les populations.

Mais si ces maladies sont endémiques à ces écosystèmes de bas-fonds, elles sont aujourd'hui en nette régression car les basfonds sont défrichés et aménagés avec moins de poches d'eau dormante qui constituaient des gîtes pour les vecteurs de ces maladies. Quant aux gastro-entérites et le choléra, elles sont souvent liées au manque d'hygiène des ménages et non à la fréquentation des basfonds mais, la prévalence de celles-ci aussi recule dans les exploitations avec les actions de sensibilisation dans toute la zone.

\section{Conclusion}

Au terme de cette étude, les résultats ont montré que l'exploitation agricole des basfonds est une source supplémentaire de revenus pour les producteurs, mais aussi des menaces pour la santé des populations car elle entraîne une recrudescence des Maladies Liées à l'Eau (MLE) notamment les gastro-entérites, les bilharzioses et l'ulcère de Buruli dans les ménages si des précautions hygiéniques ne sont pas prises. Par contre, l'exploitation agricole des bas-fonds n'augmente pas la prévalence du paludisme dans les ménages. De façon générale, ces maladies impactent négativement sur la productivité des ménages (perte financière et perte de temps de travail) comparativement aux ménages exploitant exclusivement les plateaux.

En fonction des réalités du terrain, les actions à mener pour une gestion durable et une meilleure valorisation des bas-fonds sont entre autres : l'amélioration de la maîtrise de l'eau au niveau des bas-fonds, l'adoption des systèmes intégrés combinant la valorisation agricole, pastorale et piscicole. Mais avant toute chose, il faut que les exploitants prennent conscience des maladies liées à l'eau surtout l'ulcère de Buruli qui a des conséquences considérables sur leur santé. Ainsi, la présente étude mérite d'être poursuivie pour comprendre les stratégies à mettre en œuvre par les ménages pour éviter ces maladies ou pour surmonter les périodes de crise sanitaire dues aux Maladies Liées à l'Eau (MLE). 


\section{CONFLIT D'INTERETS}

Il n'existe pas de conflit d'intérêts entre les différents auteurs de cet article.

\section{CONTRIBUTIONS DES AUTEURS}

BACS a conduit les travaux de recherches sur le terrain avec l'appui technique de HBM puis, à la rédaction de l'article. Les contributions de BACS et HBM peuvent être estimées respectivement à $40 \%$ et $50 \%$. Les autres auteurs (BSP et AKE) ont contribué à la lecture; la mise en forme et l'actualisation des références bibliographiques ont été assurées par AMV, soit autour de 5\%.

\section{RÉFÉRENCES}

Abou M, Yabi I, Yolou I, Ogouwale E. 2018. Caractérisation des systèmes de production sur les sites d'aménagements hydro-agricoles dans le doublet DangboAdjohoun au sud du Bénin. Int. J. Biol. Chem. Sci., 12(1): 462-478. DOI: https://dx.doi.org/10.4314/ijbcs.v12i1.36

Agbodjogbé JG. 2008. Impacts économiques, sanitaires et environnementaux de la mise en valeur du bas-fond Aïzè commune de OUINHI, département du ZOU. Thèse d'ingénieur agronome (AGE/FSA/UAC); $102 p+$ Annexes.

Agbossou E, Ahamidé B, Hounsou BM, Dossouhoui F. 2005. Rapport d'étude socio-économique de l'aménagement de 100 ha de bas-fond dans la commune de Covè. 22 p.

Aho N, Kossou DK. 1997. Précis d'agriculture tropicale, bases et éléments d'application édition Flamboyant. 464p.

Asanté $\mathrm{F}$, Asenso-Okyèrè $\mathrm{K}$, d'Almeida $\mathrm{S}$, Mwabu G, Okorosobo T. 2004. Poids économique du paludisme dans la région africaine : le cas du Ghana. In Bulletin des Maladies Transmissibles pour la Région Africaine, 2(4): 16p.

Atidegla CP, Degbo EH, Azonhe T, Agbossou KE. 2017. Impacts socioéconomiques et sanitaires de l'exploitation agricole de la plaine inondable Todé-Gbamè au Bénin. European Scientific Journal, 13(18) : 185-203.

Audibert M, Etard JF. 2003. Productive benefits after investment in health in Mali. Economic Development and Cultural Change, 51(3): 769-782.

Azonhè TH. 2009. Analyse systémique des déterminants environnementaux de la morbidité paludique et diarrhéique chez les populations du secteur agricole dans la dépression des Tchi au sud du Bénin. Thèse de doctorat unique de l'université d'Abomey-calavi ; 89p.

Badjito P. 2012. Caractérisation agro-sociosanitaire des bas-fonds de la commune d'Adja-Ouèrè (Département du plateau, Bénin). Mémoire de diplôme d'étude approfondie, FSA/UAC, 81p.

Badjito PS, Hounssou BM, Azonhe TH, Agbossou KE. 2014. Impacts sanitaires de l'exploitation agricole des bas-fonds dans la commune d'Adja-Ouèrè au Bénin. Annales des Sciences Agronomiques 18(1) : 19-29.

Biaou CF. 2012. Fonctionnement et viabilité des organisations de producteurs exploitant les bas-fonds dans les Départements de l'Atacora et de la Donga au nord-ouest du Bénin. Bulletin de la Recherche Agricole du Bénin (71): 26-37

Dagnelie P. 1973. Théories et méthodes statistiques. Applications agronomiques, tome 1, 2 ème édition, 375p.

Hounsou BM. 2003. Evaluation des aspects morpho-pédologiques et hydrologiques dans la caractérisation pour l'aménagement des bas-fonds dans le bassin versant de Tèwi : cas du bas-fond d'Adjanoudoho (commune de DassaZoumè), Mémoire de DESS/FSA/UAC, $91 \mathrm{p}$.

Iwikotan AA, Mama VJ, Houngbo E, Tente B. 2016. Exploitation des bas-fonds : un enjeu important pour le développement socioéconomique du Bénin. Annales de la 
Faculté des Lettres, Arts et Sciences Humaines Université d'Abomey-Calavi (Bénin) 3(22) : 59-73.

Jamin J-Y, Bouarfa S, Poussin J-C, Garin P. 2011. Les agricultures irriguées face à de nouveaux défis. Cahiers Agricultures, 20(1-2) : 10-15.

Jourdain D, Quang DD, Cuong TPV, Jamin JY. 2011. Différenciation des exploitations agricoles dans les montagnes du Nord du Vietnam : le rôle clé de l'accès à l'eau ? Cah Agric, 20 : 48-59.

Kinkingninhou M. 2003. Etude sociale et économique des périmètres rizicoles en vue de leur réhabilitation dans le cadre du développemùent local : cas du périmètre rizicole de Koussin Lélé dans la commune de Covè. Thèse d'ingénieur Agronome, ESAC/FSA/UAC, 137 p.

Konnon D, Sotondji SC, Adidéhou YA. 2014. Etude d'état des lieux de la filière riz au Bénin. Rapport d'étude CCR-B, 88p.

Malézieux E, Moustier P. 2005. La diversification dans les agricultures du Sud : à la croisée de logiques d'environnement et de marché. Cahiers d'Etudes et de Recherches Francophones/Agricultures, 14(4) : 375382.

Mama TN. 2013. Etude diagnostique d'une exploitation hydro-agricole: cas du périmètre rizicole de Bamè dans la commune de Zagnanado. Mémoire de Licence, ENSTA, 56 p.

Orsot MN, Meite A, N'guessan NA, Dje NN, Ouattara M, Coulibaly JT, Assare RK, Diakité NR, Mama AD, Dje LMA, N'Goran EK. 2018. Detailed distribution of schistosomiasis and soil-transmitted helminthiasis among schoolchildren in the Bélier and Marahoué regions, central Côte d'Ivoire : a tool for an efficient control. Int. J. Biol. Chem. Sci., 12(4): 1532-1542.

DOI: https://dx.doi.org/10.4314/ijbcs.v12i4.1

Tizio S. 2004. Etat de santé et systèmes de soins dans les pays en développement : $\mathrm{La}$ contribution des politiques de santé au développement durable. Revue Mondes en Développement, 32(127) : 17.

Son G, Kiogo R, Ye GS. 2016 Analyse des systèmes de production de l'oignon bulbe autour du barrage de Goinré dans la province du Yatenga au Nord du Burkina Faso. Int. J. Biol. Chem. Sci., 10(3): 11731183.

DOI: http://dx.doi.org/10.4314/ijbcs.v10i3.22

Vitchoekè J-M. 2006. Evaluation socioéconomique et sanitaire de la mise en valeur du bas-fond Ouantégo de la commune de Covè. Thèse d'ingénieur agronome, UAC/FSA/AGRN, 101p. 